\title{
CHIPS: an Extensible Toolbox for Cellular and Hemodynamic Two-Photon Image Analysis
}

\author{
Barrett, Matthew J P ; Ferrari, Kim David ; Stobart, Jillian L ; Holub, Martin ; Weber, Bruno
}

\begin{abstract}
Two-photon microscopy is able to produce exquisite and informative functional images of cells and blood vessels in living animals or tissue, 1 especially when combined with genetically-encoded indicators of cellular activity. 2 Since these imaging techniques typically produce too much data to analyse manually, a number of automated or semi-automated image analysis approaches have been developed. However, even aside from the algorithms themselves, many factors can complicate analysis workflows.
\end{abstract}

DOI: https://doi.org/10.1007/s12021-017-9344-y

Posted at the Zurich Open Repository and Archive, University of Zurich

ZORA URL: https://doi.org/10.5167/uzh-146000

Journal Article

Accepted Version

Originally published at:

Barrett, Matthew J P; Ferrari, Kim David; Stobart, Jillian L; Holub, Martin; Weber, Bruno (2018). CHIPS: an Extensible Toolbox for Cellular and Hemodynamic Two-Photon Image Analysis. Neuroinformatics, 16(1):145-147.

DOI: https://doi.org/10.1007/s12021-017-9344-y 


\section{CHIPS: an extensible toolbox for cellular and hemodynamic two-photon image analysis}

Matthew J.P. Barrett ${ }^{1, *}$, Kim David Ferrari ${ }^{1}$, Jillian L. Stobart ${ }^{1}$, Martin Holub ${ }^{1}$ and Bruno Weber ${ }^{1}$

1'Institute of Pharmacology and Toxicology, University of Zurich and Neuroscience Center Zurich, Winterthurerstrasse 190, 8057 Zürich, Switzerland.

*Corresponding author. Email: mbarrett@pharma.uzh.ch; Phone: +41 446356086. 
Two-photon microscopy is able to produce exquisite and informative functional images of cells and blood vessels in living animals or tissue, ${ }^{1}$ especially when combined with geneticallyencoded indicators of cellular activity. ${ }^{2}$ Since these imaging techniques typically produce too much data to analyse manually, a number of automated or semi-automated image analysis approaches have been developed. However, even aside from the algorithms themselves, many factors can complicate analysis workflows.

For example: 1) it can be tedious to extract and format the metadata required by the algorithm from the raw images; 2 ) users may want to perform one or more pre-processing steps, such as motion correction or spectral unmixing, before starting the primary analysis; 3 ) even when code is available for a published algorithm, it may not be supplied in a form that can be executed easily by users with limited programming experience, and it may not run on different platforms or in different software versions; 4) users may want to run different algorithms for the same type of analysis (e.g. for measuring activity in different cell types), but not maintain multiple disparate processing pipelines; 5) the process of optimising analysis parameters, plotting, and data output can be tedious; and 6) processing groups of similar images may be difficult or inefficient. With these factors in mind, we developed CHIPS (Cellular and Hemodynamic Image Processing Suite), an open-source toolbox, to streamline image analysis pipelines.

CHIPS integrates a number of algorithms, both novel and existing, into a complete image processing pipeline, and is applicable to a range of cellular and hemodynamic image types (Fig. 1a). For example, CHIPS is able to analyse: velocity in blood vessels based on the movement of red blood cells along a line scan; ${ }^{3,4}$ diameter of blood vessels based on either line scans across the vessel or full frame images of the vessel cross section; 5 and activity in cells using a wide range of indicators. $6,7,8$

\footnotetext{
${ }^{1}$ Shih, A. Y., Driscoll, J. D., Drew, P. J., Nishimura, N., Schaffer, C. B., \& Kleinfeld, D. (2012). Two-photon microscopy as a tool to study blood flow and neurovascular coupling in the rodent brain. Journal of Cerebral Blood Flow and Metabolism, 32(7), 1277-1309, doi:10.1038/jcbfm.2011.196.

${ }^{2}$ Lin, M. Z., \& Schnitzer, M. J. (2016). Genetically encoded indicators of neuronal activity. Nature Neuroscience, 19(9), 1142-1153, doi:10.1038/nn.4359.

3 Drew, P. J., Blinder, P., Cauwenberghs, G., Shih, A. Y., \& Kleinfeld, D. (2010). Rapid determination of particle velocity from space-time images using the Radon transform. Journal of Computational Neuroscience, 29(1-2), 5-11, doi:10.1007/s10827-009-0159-1.

${ }^{4}$ Kim, T. N., Goodwill, P. W., Chen, Y., Conolly, S. M., Schaffer, C. B., Liepmann, D., et al. (2012). Linescanning particle image velocimetry: an optical approach for quantifying a wide range of blood flow speeds in live animals. PloS One, 7(6), e38590, doi:10.1371/journal.pone.0038590.

${ }^{5}$ Gao, Y. R., \& Drew, P. J. (2014). Determination of vessel cross-sectional area by thresholding in Radon space. Journal of Cerebral Blood Flow and Metabolism, 34(7), 1180-1187, doi:10.1038/jcbfm.2014.67. ${ }^{6}$ Ellefsen, K. L., Settle, B., Parker, I., \& Smith, I. F. (2014). An algorithm for automated detection, localization and measurement of local calcium signals from camera-based imaging. Cell Calcium, 56(3), 147-156, doi:10.1016/j.ceca.2014.06.003.

${ }^{7}$ Mukamel, E. A., Nimmerjahn, A., \& Schnitzer, M. J. (2009). Automated analysis of cellular signals from large-scale calcium imaging data. Neuron, 63(6), 747-760, doi:10.1016/j.neuron.2009.08.009.
} 


\section{Requirements}

CHIPS is implemented as a series of classes and supporting functions in MATLAB (MathWorks, Natick, MA, USA), and has been tested on computers running Windows, macOS and several Linux distributions, using MATLAB versions from R2013a. CHIPS is also expected to run in earlier MATLAB versions; however, this cannot be guaranteed since the unit testing framework did not exist prior to R2013a. Every effort has been made to eliminate the use of additional MATLAB toolboxes, but it is impractical in certain cases. In addition, while all algorithms work from R2013a, some perform better in more recent versions.

\section{Workflow Overview}

Importing images (Fig. 1b). CHIPS currently supports two primary image formats: TIF images generated by ScanImage, ${ }^{9}$ and the wide range of images compatible with the OME BioFormats framework. ${ }^{10}$ In most cases, CHIPS can import the relevant metadata from these images, removing the need for manual entry. CHIPS can also import TIF images directly, and use any other image data imported into MATLAB via existing scripts.

Pre-processing (Fig. 1c). CHIPS includes a range of pre-processing features and helper functions such as: motion correction, with either whole frame or line-by-line shifts; ${ }^{11}$ spectral unmixing; channel combinations (e.g. creating a ratio channel for FRET sensors); tools to combine, split or downsample images; and denoising. ${ }^{12,13,14}$

Processing (Fig. 1d). All algorithms execute without interaction, and many are parallelised. In some cases CHIPS offers more than one choice of algorithm for the same type of analysis.

\footnotetext{
${ }^{8}$ Stobart, J. L., Ferrari, K. D., Barrett, M. J. P., Stobart, M. J., Looser, Z. J., Saab, A. S., et al. (2016). Long-term In Vivo Calcium Imaging of Astrocytes Reveals Distinct Cellular Compartment Responses to Sensory Stimulation. Cerebral Cortex, doi:10.1093/cercor/bhw366.

${ }_{9}^{9}$ Pologruto, T. A., Sabatini, B. L., \& Svoboda, K. (2003). ScanImage: flexible software for operating laser scanning microscopes. Biomed Eng Online, 2, 13, doi:10.1186/1475-925X-2-13.

${ }^{10}$ Linkert, M., Rueden, C. T., Allan, C., Burel, J. M., Moore, W., Patterson, A., et al. (2010). Metadata matters: access to image data in the real world. Journal of Cell Biology, 189(5), 777-782, doi:10.1083/jcb.201004104.

${ }^{11}$ Dombeck, D. A., Khabbaz, A. N., Collman, F., Adelman, T. L., \& Tank, D. W. (2007). Imaging large-scale neural activity with cellular resolution in awake, mobile mice. Neuron, 56(1), 43-57, doi:10.1016/j.neuron.2007.08.003.

12 Dabov, K., Foi, A., Katkovnik, V., \& Egiazarian, K. (2007). Image denoising by sparse 3-D transformdomain collaborative filtering. Ieee Transactions on Image Processing, 16(8), 2080-2095, doi:10.1109/Tip.2007.901238.

${ }_{13}$ Danielyan, A., Wu, Y. W., Shih, P. Y., Dembitskaya, Y., \& Semyanov, A. (2014). Denoising of two-photon fluorescence images with block-matching 3D filtering. Methods, 68(2), 308-316, doi:10.1016/j.ymeth.2014.03.010.

14 Makitalo, M., \& Foi, A. (2013). Optimal Inversion of the Generalized Anscombe Transformation for Poisson-Gaussian Noise. Ieee Transactions on Image Processing, 22(1), 91-103, doi:10.1109/Tip.2012.2202675.
} 
Plotting and data output (Fig. 1e). CHIPS offers a number of inbuilt plots and plotting options for quickly and easily visualising the data from each algorithm. CHIPS also includes a GUI to simplify the process of parameter optimisation. Users can access the data produced by CHIPS for further analysis in MATLAB, to produce custom figures, or to export to a comma separated values (CSV) file for use in external programs.

Groups of images. CHIPS streamlines analysing groups of images in several ways. For example, it is straightforward to process groups of nearly identical images (e.g. repeated trials from the same location) with the same parameters, and groups of similar images (e.g. the same protocol applied to different locations) that require slightly different parameters. CHIPS also includes features to analyse different parts of the same image in different ways, such as measuring activity of a cell and diameter of a blood vessel simultaneously.

\section{Implementation and Performance}

CHIPS has been designed to minimise the level of expertise required to operate, while also allowing flexibility for more advanced users. For example, most functions can be accessed via simple commands that prompt for input when needed; however, all functions can be integrated into custom scripts or functions that run without interaction. Documentation and examples for CHIPS are accessible through the standard MATLAB functions help and doc.

Given the large file size of many images, CHIPS aims to use memory conservatively, for example via handle classes that minimise duplication of data in memory. CHIPS also supports parallel processing in several ways, such as parallelisation within individual algorithms or processing different images in parallel (Fig. 1d).

CHIPS has been built using a modular framework to permit and encourage extensions. This makes it relatively straightforward for developers and collaborators to integrate new types of analysis or new algorithms for existing analysis types.

\section{Summary}

We present CHIPS, an open-source Cellular and Hemodynamic Image Processing Suite designed to analyse functional images of cells and blood vessels, primarily from two-photon microscopy. CHIPS is a set of classes and functions for MATLAB that integrates a number of algorithms, both novel and existing, into a complete image processing pipeline. CHIPS is simple to use for those without programming experience, but can also be called with custom-written scripts for more power and flexibility. CHIPS has also been designed using a modular framework to permit and encourage future extensions. 


\section{Information Sharing Statement}

The source code, along with a self-contained MATLAB toolbox, documentation, and examples, is hosted on GitHub (https://github.com/EIN-lab/CHIPS) and is available under the GNU General Public Licence v3.0.

\section{Acknowledgements}

The authors are grateful to the many colleagues and collaborators who have provided feedback, suggestions and testing during the development process; to Rachel Barrett for assistance with documentation; and to the many developers who have made versions of their code and/or algorithms available. MJPB and JLS received funding from the University of Zurich Forschungskredit. JLS received funding from the Heart and Stroke Foundation of Canada. BW is a member of the University of Zurich Clinical Research Priority Program on Molecular Imaging.

\section{Compliance with Ethical Standards}

The authors declare that they have no conflict of interest. 
(a)

Analysis Types

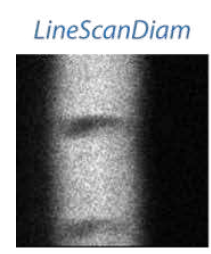

LinescanVel

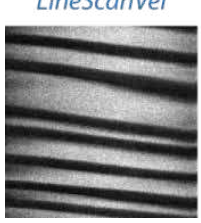

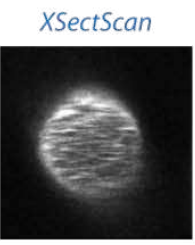

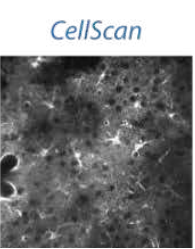

(b)

(c)

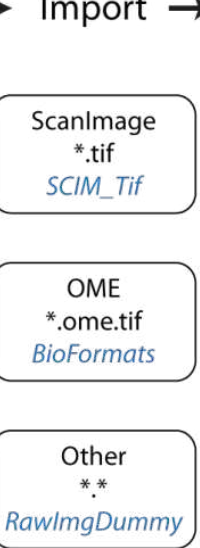

(d)

Pre-process

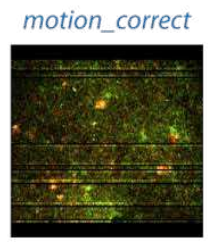

unmix_chs

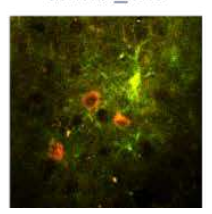

(e)

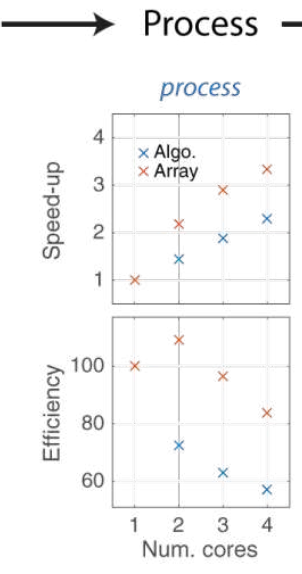

Plot + Output

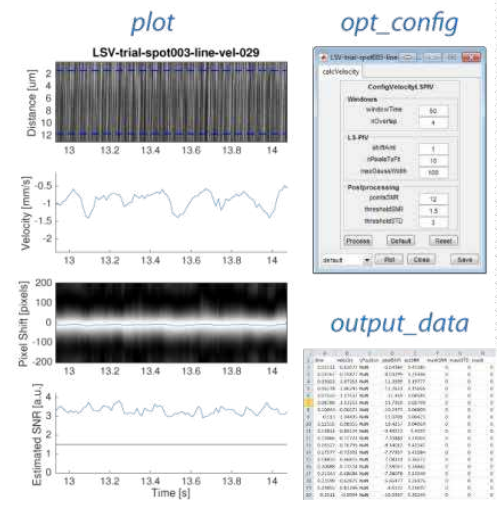

Fig. 1. Overview of CHIPS. (a). Examples of the available types of analysis. Measurement of: blood vessel diameter from a line scan across the vessel (top left); blood vessel diameter from full frame images of the vessel cross section (top right); red blood cell velocity from a line scan along the vessel centreline (bottom left); cellular activity (bottom right). (b) Image formats CHIPS can import, showing the typical file extension. (c) Examples of the pre-processing features available: motion correction (top, line-by-line correction showing artefacts from large movements); spectral unmixing of fluorophores to reduce the influence of unwanted bleedthrough (bottom). (d) Examples of the speed-up (top) and parallelisation efficiency (bottom) when parallelisation is used within an algorithm (Algo., blue) or when processing an array of images (red). The algorithm in this example was our implementation of Thresholding in Radon Space (Gao and Drew 2014). (e) Example output available after processing a LineScanVel object: the default plot (left); the GUI to assist with parameter optimisation (top right); the data exported to CSV format (bottom right). In all panels text in blue italics represents the relevant class (beginning with uppercase) or method names (beginning with lowercase) in CHIPS. 